\title{
Scope of resident ophthalmology consultation service and patient follow-up rates at a level I trauma center in Brooklyn, New York
}

This article was published in the following Dove Press journal:

Clinical Ophthalmology

29 March 2013

Number of times this article has been viewed

\section{Allison E Rizzuti \\ Marianna Vastardi \\ Mohammedyusuf Hajee \\ Douglas R Lazzaro}

Departments of Ophthalmology, SUNY Downstate Medical Center, Kings County Hospital Center, Brooklyn, NY, USA
Correspondence: Douglas R Lazzaro 450 Clarkson Avenue, Box 58,

Brooklyn, NY I I 203, USA

$\mathrm{Tel}+\mathrm{I} 7182455460$

Fax +I 7I82455332

Email douglas.lazzaro@downstate.edu
Background: The purpose of this study was to investigate the most common reasons for urgent ophthalmology consultations in both the emergency room and inpatient settings at a large public hospital served by a busy ophthalmology residency program, and to track patient follow-up rates.

Methods: We reviewed the medical records of all patients evaluated by the ophthalmology consultation service at Kings County Hospital Center from June 2003 to October 2005 using a retrospective hospital-based study design. We categorized emergency room patients and inpatients into traumatic and nontraumatic subgroups, and looked at diagnoses and patient demographics, as well as follow-up patterns for emergency room consultations.

Results: In total, 743 patients were evaluated; 436 (59\%) were emergency room patients and 307 (41\%) were inpatients. Consultation for traumatic eye injury was provided for 399 patients (54\%), accounting for $284(65 \%)$ of the emergency room consults and $115(37.5 \%)$ of the inpatient consults. The most common reason for inpatient consultation was to rule out ocular manifestations of systemic disease (57 patients, 29.7\%), while the most common final diagnosis for trauma inpatient consultation was orbital wall fracture (59 patients, $51.3 \%$ ). In total, 262 patients $(60 \%)$ in the emergency room consultation group returned for follow-up care; $162(57 \%)$ of the trauma patients followed up and $100(66 \%)$ of the nontrauma patients followed up.

Conclusion: This study provides a comprehensive analysis of the eye conditions and follow-up rates evaluated by the ophthalmology service at Kings County Hospital Center. By evaluating the follow-up patterns of these patients, we may be able to alter patient counseling to increase patient compliance.

Keywords: ophthalmology, residency program, urgent consultation, inpatients, outpatients, follow-up

\section{Introduction}

It is estimated that over 2.5 million eye injuries occur in the United States every year, many of which can lead to permanent vision loss. ${ }^{1}$ While certain ophthalmologic conditions can be managed solely by emergency room and primary care clinicians, there are a number of entities that require urgent evaluation by an ophthalmologist. Globe trauma, chemical injury, acute angle-closure glaucoma, arteritic ischemic optic neuropathy, and central retinal artery occlusion are some of the diagnoses that warrant immediate ophthalmic care or intervention. Many of the signs and symptoms of these acute vision-threatening diagnoses are also frequently seen in patients with more benign conditions. Eye pain, redness, and blurry vision are common ophthalmic complaints, and may be seen in urgent as well as nonurgent cases, and it is often only after a complete 
ophthalmic examination that the urgency can be ascertained. As a result, ophthalmologists are consulted urgently for a wide variety of signs, symptoms, and suspected diagnoses.

Kings County Hospital Center (KCHC) in Brooklyn, New York City, operates a large public ophthalmology clinic and consultation service, which is staffed by SUNY Downstate Medical Center residents and faculty alongside $\mathrm{KCHC}$ faculty. Nine of the 21 SUNY Downstate ophthalmology residents are assigned to the $\mathrm{KCHC}$ ophthalmology service. The 2012 outpatient clinic alone saw over 30,000 visits annually, making it easily the busiest public eye clinic in New York City.

The main purpose of this study was to investigate the most common reasons for urgent ophthalmology consultation in both the emergency room as well as the inpatient setting at a large urban public hospital. While we have found prior studies reporting on the epidemiology of ophthalmology consultations in an emergency room setting and in an inpatient setting (separately), we have found no studies that reported on patients seen in both settings at a single center, an analysis that would more accurately reflect the experience of a resident ophthalmology service. We also tracked the follow-up patterns of these patients in order to understand better which patients with what particular diagnoses were more likely to return for follow-up.

\section{Materials and methods}

We performed a retrospective chart review of all consecutive patients evaluated by the ophthalmology consult service at KCHC from June 2003 to October 2005. The patients either presented to the emergency department or were hospitalized inpatients (on another service) that were evaluated by the ophthalmology department the same day the consult was placed. The study was approved by the SUNY Downstate Medical Center and KCHC institutional review boards.

We obtained data regarding patient age, gender, and ocular diagnosis. Because patients may have had more than one ocular diagnosis recorded, we listed only the primary diagnosis as documented in the initial consult after it was completed. We also recorded how many of the emergency room patients returned for their follow-up outpatient clinic appointment. Follow-up information was not recorded for the inpatients, because these patients were all seen by an attending physician within 24 hours and were already hospitalized.

\section{Results}

A total of 743 patients were evaluated by the $\mathrm{KCHC}$ ophthalmology consultation service from June 2003 to
October 2005. Consultation for traumatic eye injury was provided for 399 patients (54\%). Patient age ranged from 1 day to 96 years, with a mean age of 40 years. Table 1 outlines our patient demographics according to gender, age group, and inpatient versus outpatient status.

The most frequent reason for inpatient consultation was to rule out ocular manifestations of a systemic condition (57 patients, 18.6\%). The two most common screening consultations requested were to rule out retinal hemorrhage in suspected nonaccidental trauma (12 patients, 21\% of screenings), and to rule out chorioretinitis in fungemia (six patients, $10.5 \%$ of screenings). Fifty-six patients, or $98 \%$ of all screening examinations, were negative. The one positive screening examination was to rule out Wilson's disease.

Of all inpatient consultations, there were 192 (62.5\%) nontrauma and $115(37.5 \%)$ trauma patients evaluated. The diagnoses for nontrauma inpatients are listed in Table 2. Orbital/preseptal cellulitis (16 patients, 8.3\%) and diabetic retinopathy $(10,5.2 \%)$ were the most frequent diagnoses. Diagnoses for trauma inpatients are listed in Table 3, with orbital wall fracture (59 patients, $51.3 \%$ ) and subconjunctival hemorrhage $(12,10.4 \%)$ being the most common.

Of the emergency room consultations, 152 (35\%) were nontrauma patients and 284 (65\%) were trauma patients. Diagnoses for emergency room nontrauma patients are listed in Table 4. Conjunctivitis (25 patients, 16.4\%) and glaucoma/ glaucoma suspect $(16,10.5 \%)$ were the most common. Diagnoses for emergency room trauma patients are listed in Table 5, with corneal abrasion (71 patients, 25\%) and orbital wall fracture $(56,19.7 \%)$ being the most common.

In total, $262(60 \%)$ of the patients evaluated as emergency room consults returned for their follow-up appointment. Follow-up rates by gender and age group are outlined in Table 6. Follow-up according to most frequent diagnoses

Table I Age and gender of inpatients and emergency room patients

\begin{tabular}{llll}
\hline & Inpatient (\%) & Outpatient (\%) & Total (\%) \\
\hline $\begin{array}{l}\text { Total } \\
\text { Age, years }\end{array}$ & 307 & 436 & 743 \\
$<10$ & $44(14 \%)$ & $37(8.5 \%)$ & $81(11 \%)$ \\
$10-19$ & $30(9.8 \%)$ & $49(11 \%)$ & $79(11 \%)$ \\
$20-39$ & $73(24 \%)$ & $148(34 \%)$ & $221(30 \%)$ \\
$40-59$ & $90(29 \%)$ & $143(33 \%)$ & $233(31 \%)$ \\
$60-74$ & $48(16 \%)$ & $45(10 \%)$ & $93(12.5 \%)$ \\
$75+$ & $22(7.1 \%)$ & $14(3.2 \%)$ & $36(4.8 \%)$ \\
Gender & & & $424(57 \%)$ \\
Males & $175(57 \%)$ & $249(57 \%)$ & $319(43 \%)$ \\
Females & $132(43 \%)$ & $187(43 \%)$ & \\
\hline
\end{tabular}


Table 2 Inpatient diagnoses (nontraumatic)

\begin{tabular}{ll}
\hline Diagnosis & $\begin{array}{l}\text { Patients (each } \\
\text { diagnosis, n) }\end{array}$ \\
\hline Screening examination & 57 \\
Cellulitis (orbital or preseptal) & 16 \\
Diabetic retinopathy & 10 \\
Cranial nerve palsy, glaucoma/glaucoma suspect & 9 \\
Optic neuritis & 8 \\
CNS/orbital neoplasm, conjunctivitis & 6 \\
Blepharitis, dry eye syndrome & 5 \\
CMV retinitis, herpes zoster ophthalmicus, & 4 \\
refractive error, HIV retinopathy & \\
Cataract, corneal lesion, temporal arteritis, hyphema, & 3 \\
retinal detachment, hypertensive retinopathy, & \\
posterior vitreous detachment, uveitis/iritis & 2 \\
Phthisis bulbis & \\
Exotropia, episcleritis, endophthalmitis, dacryocystitis, & I \\
globe dislocation, lacrimal fossa abscess, amaurosis \\
fugax, drug reaction, spasmus nutans, encephalitis, \\
sickle retinopathy, CRAO, CRVO, lupus retinopathy, \\
transient visual disturbance, pterygium, chemosis, eye \\
pain unknown origin
\end{tabular}

Abbreviations: CMV, cytomegalovirus; CNS, central nervous system; HIV, human immunodeficiency virus; CRAO, central retinal artery occlusion; CRVO, central retinal vein occlusion.

was $72 \%$ for corneal abrasions, $53 \%$ for orbital fractures, $64 \%$ for traumatic iritis, $56 \%$ for conjunctivitis, and $41 \%$ for corneal foreign body.

\section{Discussion}

This study provides an analysis of 743 patients seen by the $\mathrm{KCHC}$ ophthalmology consult service over a two-year period, and provides a snapshot of the patient population seen by a busy ophthalmology residency program at a large public level 1 trauma center. We have provided data on emergency room patients as well as admitted inpatients in order to reflect the most common reasons for ophthalmology consultation as accurately as possible.

Table 3 Inpatient diagnoses (traumatic)

\begin{tabular}{ll}
\hline Diagnosis & $\begin{array}{l}\text { Patients (each } \\
\text { diagnosis, n) }\end{array}$ \\
\hline Orbital wall fracture & 59 \\
Subconjunctival hemorrhage & 12 \\
Ruptured globe & $\mathrm{II}$ \\
Periorbital contusion & $\mathrm{I} 0$ \\
Hyphema & 5 \\
Eyelid laceration/canalicular tear & 4 \\
Corneal abrasion, traumatic iritis, normal eye & 3 \\
examination after trauma & \\
Retinal detachment, periorbital hematoma, cranial & $\mathrm{I}$ \\
nerve palsy, abnormally shaped pupil, retinal & \\
hemorrhage, normal eye examination after trauma & \\
\hline
\end{tabular}

Table 4 Emergency room diagnoses (nontraumatic)

\begin{tabular}{|c|c|}
\hline Diagnosis & $\begin{array}{l}\text { Patients (each } \\
\text { diagnosis, } n \text { ) }\end{array}$ \\
\hline Conjunctivitis & 25 \\
\hline Glaucoma/glaucoma suspect & 16 \\
\hline Uveitis & 14 \\
\hline Cellulitis & 7 \\
\hline Corneal disorder, posterior vitreous detachment & 6 \\
\hline $\begin{array}{l}\text { Chalazion/hordeolum, pinguecula/pterygium, } \\
\text { vitreous hemorrhage, subjective visual disturbance, } \\
\text { normal eye examination }\end{array}$ & 5 \\
\hline $\begin{array}{l}\text { HSV keratitis, blepharitis, diabetic retinopathy, } \\
\text { temporal arteritis, retinal detachment/tear }\end{array}$ & 4 \\
\hline $\begin{array}{l}\text { Blind painful eye, cataract, contact lens overwear, } \\
\text { dry eye syndrome, endophthalmitis, RVO }\end{array}$ & 3 \\
\hline $\begin{array}{l}\text { Episcleritis, cranial nerve palsy, idiopathic } \\
\text { intracranial hypertension }\end{array}$ & 2 \\
\hline $\begin{array}{l}\text { Hypertensive retinopathy, sickle retinopathy, } \\
\text { hyphema, keratoconus, scleritis, CNS neoplasm, } \\
\text { papilledema, thyroid eye disease, uveitis-glaucoma- } \\
\text { hyphema syndrome }\end{array}$ & I \\
\hline
\end{tabular}

Abbreviations: CNS, central nervous system; HSV, herpes simplex virus; RVO, retinal vein occlusion.

As a level 1 trauma center in an urban community, eye trauma was (not unexpectedly) one of the most common reasons for ophthalmologic consultation, especially in the emergency room setting, where a large majority $(65 \%)$ of ophthalmology consults were for traumatic eye injury. Of all the traumatic injuries seen by our consultation service (both inpatient and outpatient), orbital wall fracture was the most frequent diagnosis. There were 115 orbital wall fractures evaluated over the two-year period, accounting for $29 \%$ of traumatic consults and $1.5 \%$ of total consultations. This number is likely an underestimate, because it is probable that patients with more extensive eye injuries (ie, globe rupture, hyphema) may have had orbital wall fracture listed as a

Table 5 Emergency room diagnoses (traumatic)

\begin{tabular}{ll}
\hline Diagnosis & $\begin{array}{l}\text { Patients (each } \\
\text { diagnosis, } \mathbf{n})\end{array}$ \\
\hline Corneal abrasion & 71 \\
Orbital wall fracture & 56 \\
Traumatic iritis & 46 \\
Corneal/conjunctival foreign body & 29 \\
Chemical/thermal injury & 21 \\
Eyelid/canalicular laceration & 17 \\
Periorbital contusion & 18 \\
Normal eye examination & 7 \\
Subconjunctival hemorrhage & 6 \\
Hyphema & 3 \\
Conjunctival laceration, vitreous & 2 \\
hemorrhage, posterior vitreous detachment & \\
Periorbital hematoma, ruptured globe, & 1 \\
conjunctivitis, dry eye syndrome & \\
\hline
\end{tabular}


Table 6 Emergency room follow-up

\begin{tabular}{lll}
\hline & Followed up & Not followed up \\
\hline All outpatients & $262(61 \%)$ & $174(39 \%)$ \\
Trauma & $162(57 \%)$ & $122(43 \%)$ \\
Nontrauma & $100(66 \%)$ & $52(34 \%)$ \\
Gender & & \\
$\quad$ Males & $143(57 \%)$ & $106(43 \%)$ \\
$\quad$ Females & $119(64 \%)$ & $68(36 \%)$ \\
Age, years & & \\
$<10$ & $21(57 \%)$ & $16(43 \%)$ \\
$10-19$ & $28(57 \%)$ & $21(43 \%)$ \\
$20-39$ & $79(53 \%)$ & $69(47 \%)$ \\
$40-59$ & $92(64 \%)$ & $51(36 \%)$ \\
$60-74$ & $31(69 \%)$ & $14(31 \%)$ \\
$75+$ & $11(79 \%)$ & $3(21 \%)$ \\
\hline
\end{tabular}

secondary diagnosis, and were intentionally not recorded in our data.

In an epidemiological analysis of eye injuries evaluated in emergency rooms in the United States by McGwin et al, ${ }^{2}$ the most frequent type of eye injury was superficial injury of the eye and adnexa, occurring in $41.6 \%$. Superficial eye injury (corneal abrasions and corneal foreign bodies) accounted for only $23 \%$ of our emergency room patients, but it is likely that a significant number of patients with superficial eye injury were managed by the emergency room staff and did not require ophthalmology consultation. McGwin et al listed orbital floor fracture as one of their least common forms of injury, occurring in only $1.3 \%$, and did not specifically address fractures of the other orbital walls. Because we included fractures of the medial wall, lateral wall, and roof of the orbit, the number of fractures we managed was significantly higher. It should be noted that not all orbital wall fractures are seen by the ophthalmology department. Depending on the extent of the injury or coexisting pathology, the oral maxillofacial service or otolaryngology services may have been consulted instead.

Inpatient consultations are common at $\mathrm{KCHC}$, the most frequent being to screen patients for ophthalmic manifestations of systemic conditions, and accounted for $18.6 \%$ of all inpatient consultations. This is significantly lower than the figure reported by Carter et $\mathrm{al}^{3}$ who analyzed all inpatient ophthalmology consultations seen at UCLA Medical Center over a 7 -year period and found that screening examinations accounted for $28.7 \%$ of all examinations. Our most common reason for screening consultation was to evaluate a child for retinal hemorrhage in the setting of suspected child abuse. None of these patients had a positive screening examination, and were not included in the traumatic eye injury subgroup, but they nonetheless attest to the high volume of trauma cases seen at our center.
The most common screening consultation, as reported by Carter et al, was to rule out fungal endophthalmitis, while evaluating a child for retinal hemorrhage was less common (approximately 2.1\%).

It is interesting to note that $98 \%$ of all $\mathrm{KCHC}$ screening examinations were negative for the suspected condition. While urgent screening examinations may be clearly indicated in certain circumstances, especially in the setting of child abuse, other conditions can likely wait until after discharge, and would be better performed in the outpatient setting if compliance with follow-up was guaranteed. This type of model may be more suitable in hospitals with specific personnel assigned and dedicated to 24-hour follow-up calls to discharged patients. As an example, a patient recently started on ethambutol for active tuberculosis with no ocular complaints may not need urgent bedside evaluation, and would likely benefit more from a complete slit-lamp examination and Humphrey visual field testing, which can be better provided in a complete clinical setting rather than at the bedside.

The top inpatient diagnoses evaluated by our consultation service were orbital wall fracture (19.2\% of inpatients), cellulitis $(5.2 \%)$, subconjunctival hemorrhage $(3.9 \%)$, ruptured globe $(3.6 \%)$, diabetic retinopathy $(3.3 \%)$, and periorbital contusion $(3.3 \%)$. The most common outpatient diagnoses were corneal abrasion (16.3\% of outpatients), orbital wall fracture (12.8\%), traumatic iritis (10.6\%), corneal/conjunctival foreign body $(6.7 \%)$, and conjunctivitis $(5.7 \%)$. These data are once again reflective of the high incidence of trauma at our center. While we could not find any studies that looked at inpatient and outpatient consultations at a single center, we did find papers that reported on one or the other. Carter et al found the top inpatient diagnoses at UCLA to be refractive error, rule-out fungal endophthalmitis, conjunctivitis, diabetic retinopathy, and corneal abrasion. In a study of different causes of referral to the emergency room in São Paulo General Hospital, ${ }^{4}$ only $19.2 \%$ of patients had sustained traumatic eye injuries, with the most common traumatic injury being corneal foreign body in $7.5 \%$. The most frequent emergency room diagnosis at this center was conjunctivitis, seen in $29 \%$ of patients.

The follow-up rates at our center were quite low, with $40 \%$ of patients being lost to follow-up after the initial consultation. While it is possible that some of these patients sought follow-up care elsewhere, the reality is that many patients seen at KCHC lack insurance and do not have the resources to access health care in an alternative setting. It is more likely that, after being provided with an initial treatment 
plan, patients awaited resolution without further intervention. The prospect of paying for clinic visits in addition to transportation to and from the hospital may have deterred patients from appropriate follow-up. This may be satisfactory for patients with a small corneal abrasion, but clearly could be detrimental to patients with more serious diagnoses. Of our patients with orbital wall fractures, only $53 \%$ returned for a follow-up appointment. This number seems inadequate for a condition that in certain situations could potentially lead to disfiguring enophthalmos and permanent diplopia without timely surgical repair.

By analyzing the follow-up patterns of the patients evaluated, we conclude that males, patients aged $20-39$ years, and those who had sustained traumatic injuries were less likely to return for continued care. All of our patients were contacted at least three times to reschedule their outpatient appointment, but many were lost to follow-up nonetheless. An effort should be made to identify this subgroup during the initial consultation in order to discuss the risks of inadequate follow-up in more detail. Perhaps if patients with large orbital wall fractures were informed of the risk of permanent sequelae, the follow-up rates would be higher. Also, if financial burden is a deterring factor, it should be addressed during the initial consult, so that patients are aware that follow-up care would not depend on their ability to pay.

While we may make an effort to increase the follow-up rates at our center, the knowledge that a large number of our patients will be lost to follow-up despite our efforts is important when formulating initial treatment plans. Topical steroids may not be the best treatment if intraocular pressure is not subsequently monitored, and absorbable sutures may be preferable in patients who are unlikely to return for suture removal. With this knowledge in hand, we may be better able to provide care that is most appropriate for our patient population.

In summary, this study provides a comprehensive analysis of the variety of eye conditions evaluated by the ophthalmology consult service at $\mathrm{KCHC}$ and identifies the epidemiology and follow-up patterns of our patient population. By reporting on inpatient as well as outpatient consultations, this analysis is most reflective of the experience of an ophthalmology resident at a large public urban hospital, an experience that is quite distinct from that of an ophthalmologist practicing in an office setting or a resident training in a rural environment. By evaluating the follow-up patterns of these patients, we may be able to alter our treatment and counseling at the initial patient encounter to increase return rates and provide optimal care.

\section{Disclosure}

None of the authors have any conflict of interest or financial/ proprietary interest to report in this work.

\section{References}

1. American Academy of Ophthalmology and American Society of Ocular Trauma, United States Eye Injury Registry summary report, 1998-2002. Available from: http://www.aao.org/.../Eye-Injuries-BkgrnderLongVers Final-1.pdf. Accessed March 5, 2013.

2. McGwin G Jr, Hall TA, Xie A, Owsley C. Trends in eye injury in the United States. Invest Ophthalmol Vis Sci. 2006;47(2):521-527.

3. Carter K, Miller KM. Ophthalmology inpatient consultation. Ophthalmology. 2001;108(8):1505-1511.

4. Carvalho Rde S, Jose NK. Ophthalmology emergency room at the University of Sao Paulo General Hospital: a tertiary hospital providing primary and secondary level care. Clinics (Sao Paulo). 2007;62(3): 301-308.
Clinical Ophthalmology

\section{Publish your work in this journal}

Clinical Ophthalmology is an international, peer-reviewed journal covering all subspecialties within ophthalmology. Key topics include: Optometry; Visual science; Pharmacology and drug therapy in eye diseases; Basic Sciences; Primary and Secondary eye care; Patient Safety and Quality of Care Improvements. This journal is indexed on

Submit your manuscript here: http://www.dovepress.com/clinical-ophthalmology-journal

\section{Dovepress}

PubMed Central and CAS, and is the official journal of The Society of Clinical Ophthalmology (SCO). The manuscript management system is completely online and includes a very quick and fair peer-review system, which is all easy to use. Visit http://www.dovepress.com/ testimonials.php to read real quotes from published authors. 